\title{
Industrial Energy Management System: Design of a Conceptual Framework using loT and Big Data
}

This paper was downloaded from TechRxiv (https://www.techrxiv.org).

\section{LICENSE}

CC BY 4.0

SUBMISSION DATE / POSTED DATE

$18-11-2021 / 23-11-2021$

\section{CITATION}

Ullah, Mehar; Narayanan, Arun; Wolff, Annika; Nardelli, Pedro (2021): Industrial Energy Management System: Design of a Conceptual Framework using loT and Big Data. TechRxiv. Preprint. https://doi.org/10.36227/techrxiv.17045891.v1

$\mathrm{DOI}$ 


\title{
Industrial Energy Management System: Design of a Conceptual Framework using IoT and Big Data
}

\author{
Mehar Ullah*, Arun Narayanan*, Annika Wolff*, Pedro H. J. Nardelli* \\ ${ }^{*}$ LUT University, Lappeenranta, Finland \\ Contact: mehar.ullah@lut.fi
}

\begin{abstract}
Industrial activities consume a large portion of the total energy demand worldwide, and thus, significantly contribute to greenhouse gas emissions. Hence, they face significant economic, social and environmental pressures to create energy efficient processes and systems of production and directly manage their energy consumption, looking at aspects beyond direct costs. One of the most effective ways to reduce energy consumption in the industrial sector is to implement an energy management system. Current research into Industrial Energy Management System (IEnMS) remains insufficient, and to the best of our knowledge, a holistic framework for an IEnMS using the IoT and big data does not exist. This paper provides a comprehensive systematic literature review of the existing academic publications on IEnMS. Further, the main requirements and components of an IEnMS are identified using literature. Based on these identified requirements and components, we have designed a theoretical framework for the IEnMS using IoT and big data analytics, forming a cyber-physical system. These results illustrate how the proposed framework provides an objective methodology that can be used to select the most suitable IEnMS for different industries based on their particular requirements.
\end{abstract}

Index Terms-Energy Management Systems, Big-data, IoT Energy management, Industry, Energy

\section{INTRODUCTION}

Today, climate change is an extremely important global problem that threatens the health and safety of humankind. To mitigate the issues with climate change and protect current and future generations, it is important to not only integrate renewable energy sources but to also increase the efficiency of energy usage [1]. In particular, the energy sector is undergoing a radical transformation from a fossil-fuel-dominated energy system to a "green" energy system dominated by renewable and sustainable energy sources. Further, transforming the energy usage patterns and efficiencies in industries and residential housing sectors are key steps to save energy and mitigate climate changes [2].

Industry and industrial enterprises consume the highest amount of energy; it is estimated that approximately $42.3 \%$ of the total energy produced in the world is consumed by the industrial sector [3]. Hence, it is important to formulate and implement energy efficiency and management polices that are focused on the industrial sector and its own unique problems. Industries should be encouraged to prioritize the management and operation of its own energy system as a way to guarantee its long-term sustainability in a society that is facing the impacts of climate change. In other words, we argue that industry needs to put more efforts to effectively manage its energy demand, looking at aspects beyond short-term costs.

Energy management and control is a promising approach for an industry to reduce energy consumption, related energy costs, and carbon emissions. In the $20^{\text {th }}$ century, energy costs were a small, usually neglected, aspect for the industrial companies, because energy costs were very low as compared to the production size, and greenhouse gas emissions were not a priority. However, in the $21^{\text {st }}$, energy utilization costs and energy sourcing prices in Europe increased rapidly. As a result, the industrial sector has now begun to focus on energy management: typically, a company prepares long-term energy policies to achieve specific energy usage targets and values. An Industrial Energy Management System (IEnMS) is used to implement the entire process of controlling energy consumption, reducing greenhouse gas emissions, decreasing energy usage, and achieving significant cost savings.

This contribution aims to propose a high-level architecture for IEnMS that incorporates IoT and Big Data. This is necessary because although IoT and Big Data have been considered in home energy management systems (HEMS), their implementation and applications in IEnMS are not well studied. HEMS and IEnMS have somewhat similar objectives, but they face different implementation challenges because of the differences in purposes and operational scale. Our goal is to provide a general methodology that different industries can apply when selecting the most suitable IEnMS based on their particular needs. In other words, this paper will support industries to carry out a detailed analysis of their own energy requirements and understand the key components of their energy management schemes in order to find the best match for their IEnMS. Specifically, this focuses on finding the answers of the following three questions.

1) What are the current methods used for energy management in industrial sector?

2) What are the main requirements and components of Industrial Energy Management Systems?

3) How can a high-level architecture for a Industrial Energy Management System be designed using new technologies such as IoT and big data?

The rest of the paper is organized as follows. Section II describes the background of the paper, related literature, and the identified research gap. Section III explains the Industrial Energy Management and how energy can be managed in 
industries. Section IV elucidates a more systematic approach toward industrial energy management: here, we define an explain an Industrial Energy Management System and explain in detail why a systematic approach is both necessary and beneficial for industrial energy management. In Section V, we present our proposed theoretical framework for the Industrial Energy Management System using IoT and Big Data. Section VI concludes the paper.

\section{BACKGROUND}

There is a rich literature about decision-making processes related to energy-related issues in the industrial sector, with a broad consensus regarding solutions and paths, but also some disagreements about the impacts of making radical changes. Some groups [4], [5] have considered any investments that may include Energy Management (EnM) programs to have significant financial impacts so that the effect on profitability is the key factor that influences the implementation of EnM programs. Other authors [6], [7] indicate that energy policy, price, knowledge, and mindset are energy-related decision factors that influence EnM programs. More recently, some authors [1], [8]-[10] have also shown that energy-related decisions are based on the strategic links between the organization's main business and goal with any investment. Today, the importance and necessity of implementing EnM for the efficient utilization of energy is an established fact in the industrial sector, and most companies understand that they should have an energy management system that is utilized in a proper way [11]; as a result, many companies are implementing, or have implemented, some EnM policy. Nevertheless, there is still a misunderstanding about the relation between EnM and Energy Management System (EnMS), and a detailed research agenda is required in order to better explain the difference between EnM and EnMS [12].

According to [13], EnM is defined as the practices and processes by which the company is strategically handling energy issues and management. EnMS is, in turn, the tool that is used to implement those practices and processes. Thus, for example, EnMS must be implemented to successfully establish and practice EnM practices and processes that aim at, for instance, the reduction of energy consumption, cost, and greenhouse gas emissions. This was reinforced by another study where the authors highlight that the participation of top management as well as virtually all the staff of a company in all energy-related activities are required for not only a successful EnMS implementation that ensures that best EnM practices are applied company-wide [14].

In the literature, EnM has a systematic approach that consists of practices and processes that are considered as the supporting function for the industrial EnM. However, despite the fact that EnM is currently very important, it is not fully applied in practice in the industrial sector. This is because EnM in industries has an interdisciplinary nature, involving many factors beyond economics and technology, such as social acceptance, political positions, and managerial established practices. To guarantee a successful implementation of EnM, a form of
EnMS applied to industries - Industrial EnMS (IEnMS) - is desirable so that an objective way to monitor, plan, and control energy demand and efficiency can be constructed and the planned energy positive goals can be achieved. This is the step that is usually misunderstood, and thus, not implemented adequately and correctly in majority of the industrial sector. Further IoT and Big Data are important enabling technologies that can be used for supporting complex energy management processes but their precise roles have not been clarified in the literature so far. These are the research gaps identified here and the focus of this paper. In the subsequent sections, we will first explain EnM applied to the industry, i.e., Industrial Energy Management (IEnM), and then present how an IEnMS can be used to achieve efficient IEnM.

\section{INDUSTRIAL ENERGY MANAGEMENT}

One of the indicators of the economic development is the growth in industrial sector, and thus, an associated growth in energy consumption [15]. To mitigate the negative impacts of such a growth, it is important to develop IEnM practices and processes that will use energy efficiently and control carbon emissions [3]. As a result, in recent years, many organizations have focused on energy-related issues to improve their production and operations and improve energy efficiency, thereby reducing their energy usage.

\section{A. Components of IEnM}

We have identified key components of IEnM from the literature [15]-[19]. They are as follows-planning/strategy, operation/implementation, controlling, organization, and culture (Fig. 1).

Planning/Strategy: The Planning/Strategy component represents the first phase of IEnM, and it has three parts: (1) Written long-term energy policy of the company: most companies lack written long-term energy policies and commitments and merely handle energy issues verbally. For a successful IEnM, companies should have long-term energy policies [20]. (2) Energy planning and target setting: the industry makes plans and sets future energy usage targets [21], [22]. (3) Strategic energy risk management: companies analyze any type of risk related to the energy use and propose ways to manage the risk, based on the company's predetermined financial objectives and risk tolerance [23].

Operation/Implementation: The second componentOperation/Implementation component-represents the second phase and also has three parts: (1) Implementation of energy efficiency measures: companies implement specific energy projects and energy efficiency technologies to reduce electricity consumption [24]. (2) Investment decisions on energy efficiency measure: companies conduct systematic economic calculations to calculate the return of investment [25]. (3) Energy audit: companies within the operations of an energy management constantly review the status quo and highlight energy saving potentials. Audits can be of three types. Simple audits happen quickly and only contain some interviews with the operating employees, reviews of the facilities' accounts, 


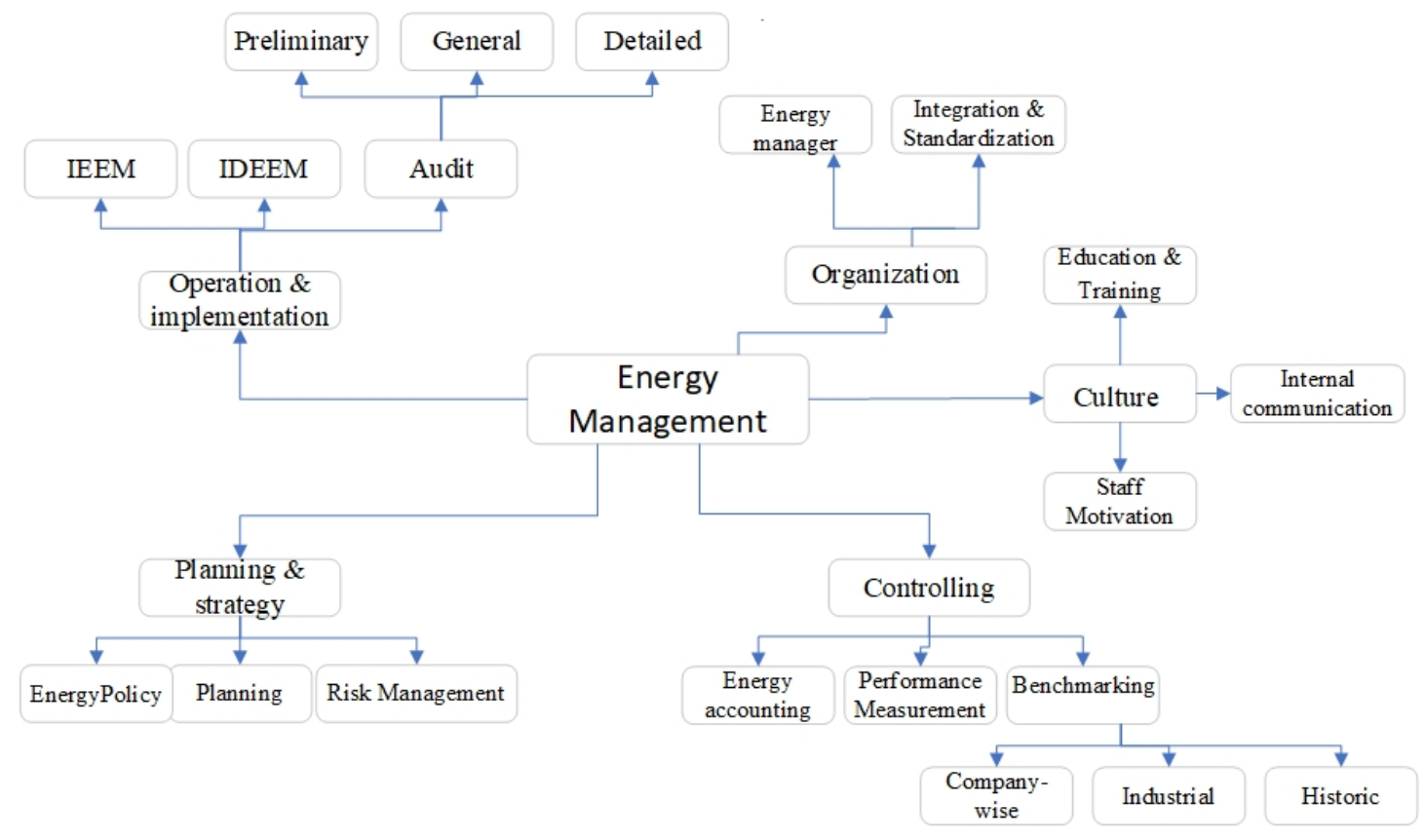

Fig. 1. Components of Energy Management System

and inspection of some operating data; this is usually conduced with a short visit by the audit personal to the facility area. General audits are a detailed expansion of the simple audit, in which detailed information is collected about the operating facility, e.g., data of energy consuming machines or systems. Detailed audits are an expansion of the general audit, which includes a dynamic model of energy-use characteristics [24].

Controlling: The third phase of EnM is represented by the third component and its three parts-energy accounting, performance measurement, and benchmarking. Energy accounting is the process of constant analyses and regular reporting of the energy consumption and measurement of the energy efficiency monitoring [26]. Performance measurement is an integral part of IEnM and defines the key performance indicators (KPIs) for energy efficiency, which describe the relationship between an activity and the required energy [17]. Energy benchmarking is an activity that is focused on energy performance and it can be defined as the method used to compare the energy efficiency between or within entities. Benchmarking is useful for achieving reductions in energy use and related costs and emissions, and there are three types of benchmarkingcompany-wise benchmarking, industrial benchmarking, and historical benchmarking. In company-wise benchmarking, the company compares its facilities and processes with the facilities and process of divisions within the company itself [27]. In industrial benchmarking, the company compares its own facilities and process with the facilities and process of other company. In historical benchmarking, the company compares the energy consumption of a process or facility with its own historical processes or facilities.

Organization: Organization, the fourth component of IEnMS, represents the fourth phase of energy management. It consists of two parts: (1) Selection of an energy manager:
The energy manager in the industry is selected based on experience and should be climate friendly. The energy manager is responsible for updating the top management about the activities and progress of the energy management, and there should be a close link between the energy manager and the top management [28], [29]. (2) Integration and standardization: According to [26], the energy management of industrial companies should be integrated with the production management processes using ICT tools and standardization. The production process and evaluation of potential energy-saving investment can be controlled by ICT tools, and the transparency of the industrial companies can be increased by the standardization.

Culture: The fifth and last component of energy management-Culture-consists of two parts: (1) Education and training: The energy manager requires personnel who either have sufficient basic education to meet the energy-usage requirements or may need to be be given training [30]. (2) Staff motivation: Industrial companies needs to motivate the staff to actively participate in improving the energy efficiency. Companies often give rewards to the technical and operational staff, which helps to sustain the momentum and improve the overall support for energy management programs [31] [26]. Staff motivation is enhanced especially by the interest of the top management in the energy management activities, and, if the communication is effective and energy management programs are standardized [27].

To achieve effective and efficient IEnM that incorporates the abovementioned components, industries must implement a company-wide energy management system, i.e., an Industrial Energy Management System (IEnMS). We now expand on the idea of an IEnMS and its key features. 


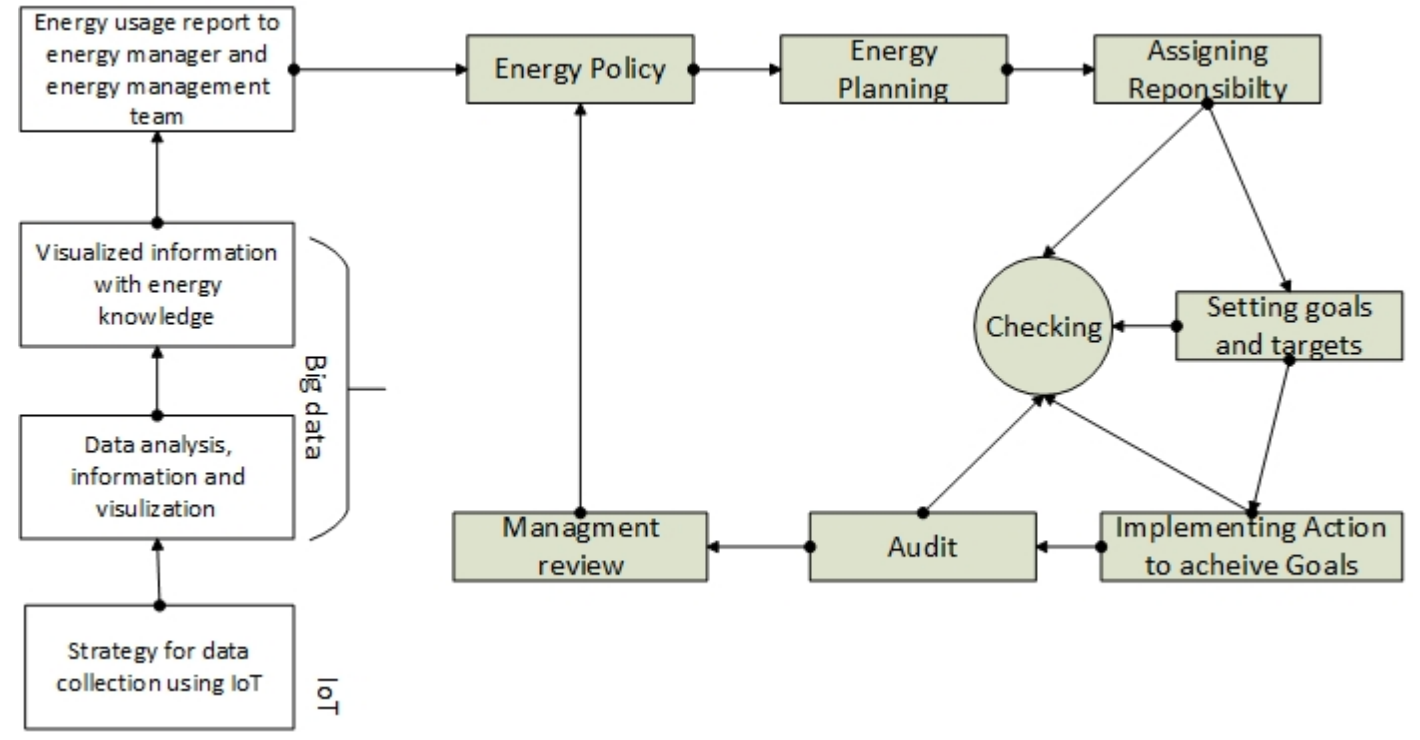

Fig. 2. Parts of an Industrial Energy Management System

\section{Industrial ENERGy MANAGEMENT System}

Industrial Energy Management System (IEnMS) refers to the technological solution designed to support the implementation of IEnM. An IEnMS monitors, controls, and optimizes the energy performance in an industrial plant and measures the consumption of energy (and potentially the generation) (Figure 2). It is also used to diagnose problems such as overconsumption and leaks across the entire plant. For energy consumers including industrial, public sectors, and commercial organizations, an IEnMS is a framework to manage their energy usage and can be defined as "a set of interrelated or interacting elements of a plan which sets an energy efficiency objective and a strategy to achieve that objective" [32]. It provides companies the opportunities to improve energy savings by adopting energy saving technologies. In most cases, a successful implementation of EnMS requires low investment cost and specialized expertise and staff training.

Industries must follow a series of defined steps to establish an effective EnMS. The main steps required are developing an energy policy and assigning responsibilities, highlighting main energy users, setting measurable goals and targets, implementing actions to achieve the goals, checking if the actions are successful, and continuous system reviews.

\section{A. Fundamentals of Industrial Energy Management Systems}

In technical terms, an energy management system (EnMS) is a computer-based system that is used to collect and measure energy data from the field, such as heating, ventilation and air-conditioning (HVAC) units, lighting system, water and gas meters installed on production line, while providing the relevant information to the user. The operation of an IEnMS can be understood as a cyber-physical system comprising the following steps.

(1) Build data collection strategy: Real-time accurate and granular data is collected along with the information about where and when the energy has been used and by which device (machine). The data is collected by installing sensors/smart meters, sub-meters, and large energy consumer devices such as HVAC (heating, ventilation and air conditioning) equipment, production line, boilers, etc. The objective of this part is to monitor the collection of real-time data and determine where the majority of the energy is being used.

(2) Transform raw energy data into useful information: In this phase, the collected data is analyzed, interpreted, and converted into useful information. Big-data software is used to easily import raw data from different machines using IoT devices and convert them into useful information in the forms of user-friendly charts. The collected raw data may be related to the production levels, weather data, and other factors that influence the energy used to generate the company's KPIs.

(3) Assign responsibility, analyze data: In this phase, the information provided must be converted into useful and meaningful reports; this is only possible by adding the information to the knowledge of the facility, which can be done by an energy manager. The role of the energy manager is to interpret the information provided by the Energy Management System and combine this information with the company's process and to set the targets accordingly.

(4) Interpret the results, and agree to an action plan: In this phase, the energy usage reports are available to the energy manager. The energy manager and energy management team start to communicate with the departments to start an energy policy and agree to an action plan.

\section{B. Benefits of Industrial Energy Management System}

IEnMS potentially enables industries to reduce its energy consumption (or improve its efficiency), reduce energy costs, and reduce carbon emissions. The main benefits of using IEnMS in companies are as follows:

Cost reduction: IEnMS is a continuous process and can bring improvements in energy performance and productivity, and reduce the energy costs over a longer period of time. 


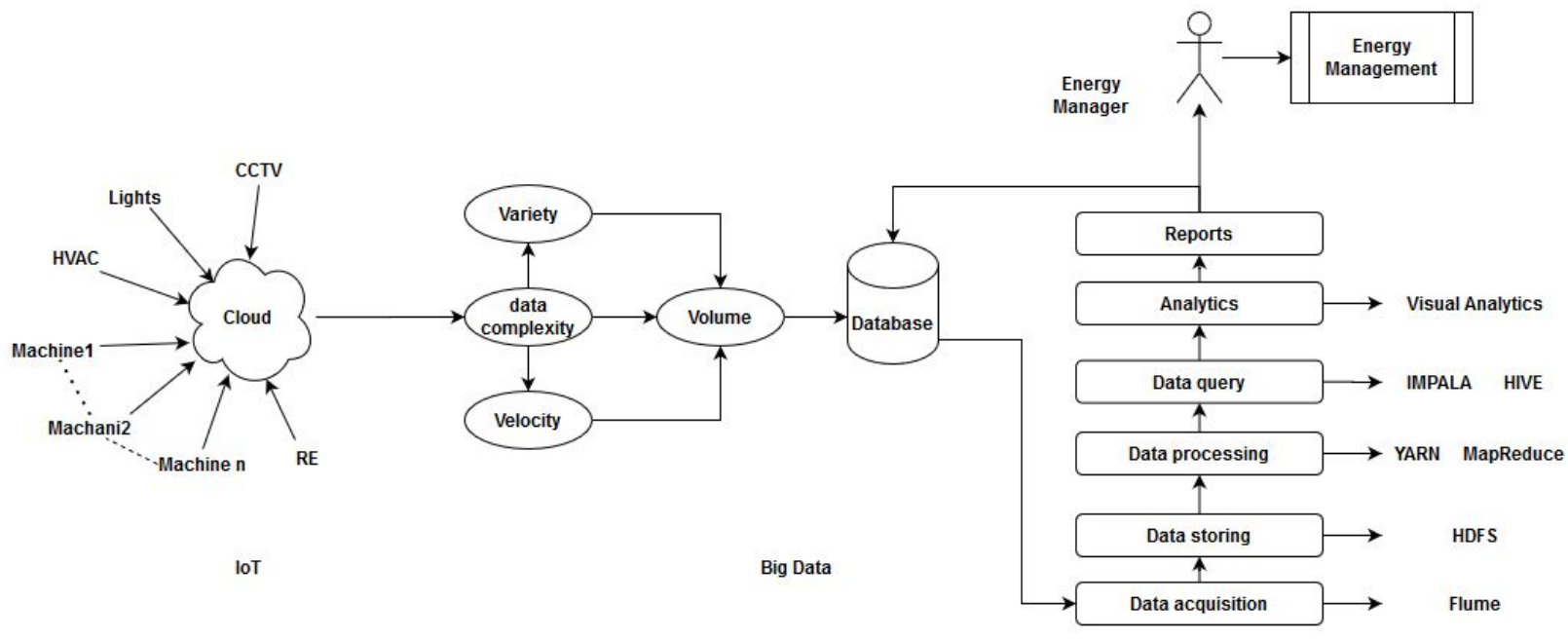

Fig. 3. Data generation using IoT and processing with Big data

Top management interest: A successful IEnMS will ensure commitment from the top management to energy efficiency.

Setting up of future targets: IEnMS gives the companies a clear picture of current energy use status, and based on the data, new future goals and targets can be set.

Organization participation: All the staff members of the organization are involved in the energy improvement process.

Energy savings: Companies can save up-to 20-30\% energy by implementing EnMS.

Carbon emission reductions: Industrial energy consumption is one of the biggest sources of carbon emission. By implementing an EnMS in industries, energy efficiency will improve, and industrial green house emission will be reduced.

Increase in machine lifespan: Using EnMS in industries can help identify and minimize machine defects that result in longer lifespan of machinery through regular maintenance.

Job creation: IEnMS can create more jobs in industries, for example, as a result of new energy efficiency projects.

Risk mitigation: Using IEnMS in industries can reduce the financial risk and energy shortage. This is because the energy demand is reduced and the fossil fuel consumption is reduced.

Improvement in company projects: Using IEnMS, companies can capture the projects' data; this will help to ensure better post-investment project performance.

Environment friendliness: Using IEnMS, the companies can systematically reduce energy usage and carbon emissions.

\section{PROPOSED HIGH LEVEL ARCHITECTURE FOR IENMS INCORPORATING IOT AND BIG DATA}

Figure 3 shows all the phases of the proposed architecture starting from data capturing to reporting. In the initial phase, data is collected using sensors and actuators from various sources in the industry that contains machines, HVAC, data generation from renewable sources (solar, wind), lighting, CCTV, and many more energy consuming devices. The huge amount of data generated by these devices is stored in a low-cost storage in the cloud. In the second phase, data acquisition, the generated Big data is based on the volume, velocity, and variety that is stored in a shared distributed fault tolerant database. The collected data is then transferred into master node(s) in the Hadoop cluster. As the data is collected from multiple heterogeneous devices, it may have different data formats and information, and data preprocessing will be required. In data preprocessing, inaccurate and incomplete data are handled, and incomplete data is either corrected or deleted. Flume is used to perform the data acquisition process. The main function of Flume is to collect, aggregate, and transfer the large amount of data to Hadoop master node.

The data received by Flume is stored in a single or multiple channel. The data is then sent to the external HDFS repository, where the data is written in a desired format using plug-in serializers. The serializers change and restructure the Flume data into the desired format. The data is pre-processed and a unified view of the data is achieved. The data is stored in the HDFS multiple clusters for processing. The HDFS clusters consists of DataNodes. The actual data and file system meta data are stored in those DataNodes. The data analysis is performed by YARN on the data stored in HDFS; these two run on the same set of nodes that allows tasks to be processed on the nodes in which the EnM data is present. Hive and Impala are the tools to perform SQL queries on data residing on HDFS. HIVE is used for data querying, to select, analyze, and to make calculations on the data of interest.

The last phase is also the most important part: data analytics. Here, the calculated data must be shared with the energy management, and especially the energy manager, so that better planning decisions can be made to achieve efficient utilization of energy, reductions in greenhouse gas emissions, increase in machine efficiency, designing of energy policy, improvements in energy planning, etc. The tool used in Hadoop for data analytics is Scalable Advanced Massive Online Analysis (SAMOA), a distributed streaming machine learning framework that consists of programming abstraction for distributed streaming algorithms for data mining and machine learning tasks. For the data visualization (graphs, reports, etc.), Tableau is used. Tableau is a common tool used for interactive data 
visualization and the sharing of information and dashboards.

\section{CONCLUSIONS}

In this paper, we have highlighted the importance of saving energy and reducing energy utilization, energy cost, and green house gas emissions in the industrial sector. We have also explained the differences between energy management and energy management systems. We have presented the key components of IEnM, identified from the literature, and indicated how they jointly work to achieve the expected targets. Further, we have explained how IEnMS can works to effectively deploy IEnM. Finally, we have designed a theoretical framework for IEnMS as a cyber-physical system enabled by IoT, Big Data, and analytics. The (big) data from different industrial machines and industrial spaces are collected as part of an IoT network using different measuring devices and transmitted to a cloud database. The proposed solution uses Hadoop for data acquisition, storing, processing, data querying, analytics, and data reporting. The information collected using real data from industrial devices can be used by the IEnMS for future energy strategy planning and establishing energy targets. We believe that if industries start using the proposed IEnMS framework, they will achieve their energy requirements, while reducing their energy usage, costs, and green house gas emissions.

\section{ACKNOWLEDGEMENTS}

This work is supported by the Academy of Finland: (a) ee-IoT n.319009, (b) EnergyNet n.321265/n.328869 and (c) FIREMAN n.326270/CHIST-ERA-17-BDSI-003; and by JAES Foundation via STREAM project.

\section{REFERENCES}

[1] A. Sa, P. Thollander, and E. Cagno, "Assessing the driving factors for energy management program adoption," Renewable and Sustainable Energy Reviews, vol. 74, no. January 2016, pp. 538-547, 2017.

[2] E. Piñero, "ISO 50001 : Setting the Standard for Industrial Energy Management," Green Manufaturing News, pp. 21-24, 2009.

[3] M. Wei, S. H. Hong, and M. Alam, "An iot-based energy-management platform for industrial facilities," Applied energy, vol. 164, pp. 607-619, 2016.

[4] D. van Soest and E. Bulte, "Does the energy-efficiency paradox exist? technological progress and uncertainty," Environmental \& Resource Economics, vol. 18, no. 1, pp. 101-112, 2001.

[5] S. T. Anderson and R. G. Newell, "Information programs for technology adoption: the case of energy-efficiency audits," Resource and Energy Economics, vol. 26, no. 1, pp. 27-50, 2004.

[6] J. Catarino, J. Henriques, and F. Egreja, "Portuguese sme toward energy efficiency improvement," Energy Efficiency, vol. 8, no. 5, pp. 995-1013, 2015.

[7] S. Sorrell, J. Schleich, S. Scott, E. O'Malley, F. Trace, U. Boede, K. Ostertag, and P. Radgen, "Reducing barriers to energy efficiency in public and private organizations," Science and Policy Technology Research (SPRU), University of Sussex, Sussex, UK, 2000.

[8] H. L. De Groot, E. T. Verhoef, and P. Nijkamp, "Energy saving by firms: decision-making, barriers and policies," Energy Economics, vol. 23, no. 6, pp. 717-740, 2001.

[9] J. Harris, J. Anderson, and W. Shafron, "Investment in energy efficiency: a survey of australian firms," Energy Policy, vol. 28, no. 12, pp. 867-876, 2000.

[10] P. Sandberg and M. Söderström, "Industrial energy efficiency: the need for investment decision support from a manager perspective," Energy policy, vol. 31, no. 15, pp. 1623-1634, 2003.

[11] M. Rudberg, M. Waldemarsson, and H. Lidestam, "Strategic perspectives on energy management: A case study in the process industry," Applied Energy, vol. 104, pp. 487-496, 2013.
[12] P. Thollander and J. Palm, "Industrial energy management decision making for improved energy efficiency-strategic system perspectives and situated action in combination," Energies, vol. 8, no. 6, pp. 5694-5703, 2015.

[13] - Improving energy efficiency in industrial energy systems: An interdisciplinary perspective on barriers, energy audits, energy management, policies, and programs. Springer Science \& Business Media, 2012.

[14] P. Peura, "From malthus to sustainable energy-theoretical orientations to reforming the energy sector," Renewable and Sustainable Energy Reviews, vol. 19, pp. 309-327, 2013.

[15] B. Purwanggono, K. Ferastra, and A. Bachtiar, "Critical success factors evaluation of the iso 50001 energy management system implementation (case study: Pt. apac inti corpora, bawen, semarang indonesia)," in IOP Conference Series: Materials Science and Engineering, vol. 598, no. 1. IOP Publishing, 2019, p. 012115.

[16] M. Schulze, H. Nehler, M. Ottosson, and P. Thollander, "Energy management in industry - A systematic review of previous findings and an integrative conceptual framework," Journal of Cleaner Production, vol. 112, no. 112, pp. 3692-3708, 2016.

[17] L. Sivill, J. Manninen, I. Hippinen, and P. Ahtila, "Success factors of energy management in energy-intensive industries: Development priority of energy performance measurement," International journal of energy research, vol. 37, no. 8, pp. 936-951, 2013.

[18] E. V. Tachmitzaki, E. A. Didaskalou, and D. A. Georgakellos, "Energy management practices' determinants in Greek enterprises," Sustainability (Switzerland), vol. 12, no. 1, 2020.

[19] A. Sa, P. Thollander, and M. Rafiee, "Industrial energy management systems and energy-related decision-making," Energies, vol. 11, no. 10, p. 2784, 2018.

[20] P. Thollander and M. Ottosson, "Energy management practices in swedish energy-intensive industries," Journal of Cleaner Production, vol. 18 , no. 12 , pp. 1125-1133, 2010.

[21] M. G. Rietbergen and K. Blok, "Setting smart targets for industrial energy use and industrial energy efficiency," Energy Policy, vol. 38, no. 8, pp. 4339-4354, 2010.

[22] R. Martin, M. Muûls, L. B. De Preux, and U. J. Wagner, "Anatomy of a paradox: Management practices, organizational structure and energy efficiency," Journal of Environmental Economics and Management, vol. 63, no. 2, pp. 208-223, 2012.

[23] S. Vasudevan and B. Higgins, "Strategic energy risk management for end users," The Journal of Structured Finance, vol. 10, no. 1, pp. 74 78, 2004.

[24] E. Abdelaziz, R. Saidur, and S. Mekhilef, "A review on energy saving strategies in industrial sector," Renewable and sustainable energy reviews, vol. 15, no. 1, pp. 150-168, 2011.

[25] R. W. Apeaning and P. Thollander, "Barriers to and driving forces for industrial energy efficiency improvements in african industries-a case study of ghana's largest industrial area," Journal of Cleaner Production, vol. 53, pp. 204-213, 2013.

[26] K. Bunse, M. Vodicka, P. Schönsleben, M. Brülhart, and F. O. Ernst, "Integrating energy efficiency performance in production managementgap analysis between industrial needs and scientific literature," Journal of Cleaner Production, vol. 19, no. 6-7, pp. 667-679, 2011.

[27] R. D. Peterson and C. K. Belt, "Elements of an energy management program," Jom, vol. 61, no. 4, pp. 19-24, 2009.

[28] D. Gordić, M. Babić, N. Jovičić, V. Šušteršič, D. Končalović, and D. Jelić, "Development of energy management system-case study of serbian car manufacturer," Energy Conversion and Management, vol. 51, no. 12, pp. 2783-2790, 2010.

[29] S. A. Ates and N. M. Durakbasa, "Evaluation of corporate energy management practices of energy intensive industries in turkey," Energy, vol. 45, no. 1, pp. 81-91, 2012.

[30] S. Suk, X. Liu, and K. Sudo, "A survey study of energy saving activities of industrial companies in the republic of korea," Journal of Cleaner Production, vol. 41, pp. 301-311, 2013.

[31] V. Blass, C. J. Corbett, M. A. Delmas, and S. Muthulingam, "Top management and the adoption of energy efficiency practices: Evidence from small and medium-sized manufacturing firms in the us," Energy, vol. 65 , pp. $560-571,2014$.

[32] V. A. da Silva Gonçalves and F. J. M.-H. dos Santos, "Energy management system iso 50001: 2011 and energy management for sustainable development," Energy Policy, vol. 133, p. 110868, 2019. 